\title{
Adverse Cutaneous Drug Reactions
}

The skin is one of the most frequently involved organs by drug side effects, with the incidence of adverse cutaneous drug reactions situated between $0.1-1 \%$ of patients during pre-marketing clinical trials, and post-marketing analyses suggest that their incidence can be as high as 1-8\% for certain types of drugs (NSAIDS, antibiotics, antiepileptics). Adverse cutaneous drug reactions are benign in nature in over $98 \%$ of cases and present most frequently as maculo-papular eruptions or urticaria. However, studies suggest that roughly a third of drug eruptions require hospital management and are considered as severe, although fortunately only $2 \%$ of cutaneous drug eruptions are really life-threatening; this is the case for Stevens-Johnson syndrome (SJS) and toxic epidermal necrolysis (TEN), acute generalized exanthematous pustulosis (AGEP), and drug rash with eosinophilia and systemic symptoms (DRESS)/drug induced hypersensitivity syndrome (DIHS). It is estimated that between 2 and $5 \%$ of all hospital costs are related to adverse drug eruptions, and cutaneous adverse drug eruptions representing $20-30 \%$ of all drug eruptions the burden of these on the health care system is considerable.

Although the pathomechanism of adverse cutaneous drug eruptions remains incompletely understood, great progress in this field of medicine has been made in the past few years. Improvements range from the clinical classification that is essential for a better understanding to the identification of genetic susceptibilities to certain drugs, and consequently the development of the first preventive genetic screening measures for selected patient groups and drug classes.

Here I will present the clinical characteristics of the different types of adverse cutaneous drug reactions and then focus on progress made in understanding the pathogenesis of two of the severe forms, notably toxic epidermal necrolysis and acute generalized exanthematous pustulosis.
Toxic epidermal necrolysis (TEN) is an immune-mediated reaction that selectively affects the skin and mucous membranes. It is triggered by drug-intake resulting in massive keratinocyte apoptosis and necroptosis within 2-4 weeks and extensive skin detachment resembling a severe burn. Compelling evidence suggests that TEN is associated with an impaired capacity to detoxify reactive intermediate drug metabolites. According to the current research data available, the immune response in TEN is dependent, at least for certain types of drugs, on HLA-restricted drug presentation, and consequently a genetic susceptibility has been reported, as evidenced by the identification of specific drug-related HLA alleles as major susceptibility genes for the development of TEN. The current model for the pathogenesis of TEN thus suggests that in an individual with particular predisposing factors (including but not always HLA type), exposure to certain types of drugs results in an immune reaction to the drug or one of its metabolites, and subsequent massive keratinocyte death mediated by FasL (CD95L) and/or granulysin and/or perforin-granzyme B and/or annexin A1. Interestingly, however, amongst individuals with a predisposing HLA type, only a minority develop TEN upon the selected drug intake (the positive predictive value of HLA-B* 15:02 for carbamazepine-induced TEN is only $5.6 \%$ ). These above facts suggest that factors other than HLA type and adaptive immunity are involved in the pathogenesis of TEN. While we and others have identified key molecular cell death mechanisms in TEN, early events driving the immune response and leading to cell death remain largely unknown.

Acute generalized exanthematous pustulosis (AGEP) is a severe adverse cutaneous drug reaction (ADR) caused mainly by antibiotics, antimalarials, and antifungals, characterized by a disseminated eruption of sterile non-follicular pustules on the background of a widespread erythematous skin eruption accompanied with fever and peripheral blood neutrophilia. The pathophysiology of AGEP remains unclear. However, as for most other ADR, AGEP is currently considered as a $T$ cell-mediated disease in which T cell- and keratinocytederived IL-8 has been proposed to be responsible for the recruitment of neutrophils to the intraepithelial pustules. Using an unbiased approach we could recently demonstrate selective and significative IL$36 \alpha$ and $y$ upregulation in lesional skin of AGEP patients when compared to MPR. Keratinocytes and macrophages were found to be the source of IL-36y in AGEP patients. In vitro, causative drugs were shown to have the ability to specifically induced IL-36y release either directly by peripheral blood monocytes, or indirectly in presence of autologous peripheral blood mononuclear cells by keratinocytes of patients with AGEP. Such culprit drug induction of IL-36y secretion in vitro was specific for AGEP and not observed for MPR. These results demonstrate a new pathogenetic mechanism for the development of AGEP whereby strong and selective IL-36y secretion by monocytes/macrophages and keratinocytes in response to culprit drug exposure drives neutrophil infiltration of the skin which is the clinical hallmark of AGEP.

Identification of key molecular signaling pathways involved in the pathogenesis of severe adverse cutaneous drug reactions such as TEN and AGEP opens new perspectives for the development of targeted treatments.

\section{Conflict of Interest}

The author declares there is no conflict of interest in connection with his contribution. Others: Lecture and/or advisory activities for: Galderma, Novartis, Janssen, Amgen, Leo Pharma, AbbVie, Eli Lilly.

Author

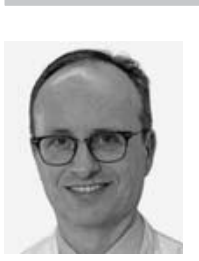

Prof. Dr., Department of Dermatology and Allergy, University Hospital of Munich, LMU, Munich, Germany 
Correspondence

Prof. Dr. Lars E. French

Direktor

Klinik und Poliklinik für Dermatologie und

Allergologie

Ludwig-Maximilians-Universität München

Frauenlobstraße 9-11

80337 München

Deutschland

lars.french@med.uni-muenchen.de

\section{References}

[1] Kuijper EC, French LE, Tensen CP et al. Clini$\mathrm{cal}$ and pathogenic aspects of the severe $\mathrm{Cu}-$ taneous adverse reaction epidermal necrolysis (EN). J Eur Acad Dermatol Venereol 2020; doi:10.1111/jdv.16339

[2] Hoetzenecker W, Nägeli M, Mehra ET et al. Adverse cutaneous drug eruptions: current understanding. Semin Immunopathol 2016; 38: $75-86$
[3] Meier-Schiesser B, Feldmeyer L, Jankovic D et al. Culprit Drugs Induce Specific IL-36 Overexpression in Acute Generalized Exanthematous Pustulosis. J Invest Dermatol 2019; 139 : 848-858 